\title{
Laser and electron deflection from transverse asymmetries in laser-plasma accelerators
}

\author{
Daniel E. Mittelberger $\odot,{ }^{*}$ Maxence Thévenet $\odot$, Kei Nakamura, Anthony J. Gonsalves, Carlo Benedetti, Joost Daniels, ${ }^{\dagger}$ \\ Sven Steinke, Rémi Lehe, Jean-Luc Vay, Carl B. Schroeder, Eric Esarey, and Wim P. Leemans \\ Lawrence Berkeley National Laboratory, Berkeley, California 94720, USA
}

(Received 20 August 2019; revised manuscript received 25 October 2019; published 23 December 2019)

\begin{abstract}
We report on the deflection of laser pulses and accelerated electrons in a laser-plasma accelerator (LPA) by the effects of laser pulse front tilt and transverse density gradients. Asymmetry in the plasma index of refraction leads to laser steering, which can be due to a density gradient or spatiotemporal coupling of the laser pulse. The transverse forces from the skewed plasma wave can also lead to electron deflection relative to the laser. Quantitative models are proposed for both the laser and electron steering, which are confirmed by particle-in-cell simulations. Experiments with the BELLA Petawatt Laser are presented which show controllable 0.1-1 mrad laser and electron beam deflection from laser pulse front tilt. This has potential applications for electron beam pointing control, which is of paramount importance for LPA applications.
\end{abstract}

DOI: 10.1103/PhysRevE.100.063208

\section{INTRODUCTION}

Laser plasma accelerators (LPAs) have been able to generate electron beams with increasing energy and quality [1-6]. Plasma waves driven by a laser pulse can sustain accelerating gradients on the order of $10-100 \mathrm{GV} / \mathrm{m}$, which allows acceleration of electrons to hundreds of $\mathrm{GeV}$ within a few meters distance. Hence, LPAs can be made more compact than conventional accelerators and are seen as potential alternatives for a number of applications, including high-energy physics [7], x-ray generation [8], ultrafast electron diffraction [9], and radiation biology [10]. While LPAs can produce electron beams with energies from the $\mathrm{MeV}$ range [11] to the multi$\mathrm{GeV}$ range [2,3] routinely, the sub-mrad pointing control that is needed for most applications is not possible without the aid of additional beam optics [12-14].

Several studies have showed that laser imperfections can alter significantly the microscopic dynamics of an LPA [13,15-17]. Reference [18] demonstrated that tilting of the laser pulse front via angular dispersion could bias the direction of the accelerated electron beams. Reference [19] showed that tilting the laser pulse front caused polarization of the $\mathrm{x}$-ray photons created by betatron oscillations, indicating an effect on the electron motion in the wake. Reference [20] showed that spatiotemporal or intensity asymmetry in the focal spot can seed a hosing instability for pulses with lengths $L \gtrsim \lambda_{p}$, where $\lambda_{p}$ is the plasma wavelength, resulting in long-wavelength oscillations of the laser transverse position. Asymmetry in the plasma instead of the laser can also result in laser deflection [21]. A quantitative understanding of the

\footnotetext{
*mittelberger1@1lnl.gov; present address: Lawrence Livermore National Laboratory, Livermore, California 94550, USA.

${ }^{\dagger}$ Present address: Monterey Bay Aquarium Research Institute, Moss Landing, California 95039, USA.

${ }^{\ddagger}$ Present address: Deutsches Elektronen-Synchrotron (DESY), Hamburg, Germany.
}

effect of laser and plasma asymmetry on electron beam quality and steering would thus be valuable for the optimization of laser systems dedicated to LPA applications. Furthermore, this mechanism could potentially be applied for electron beam pointing feedback and/or stabilization in a high repetition rate (e.g., kHz) LPA.

In this article, we propose quantitative models for the effects of laser pulse front tilt (PFT) and transverse density gradient on the laser pulse propagation and accelerated electron beam steering for the short laser pulses, $L \lesssim \lambda_{p} / 2$, typically used in LPA experiments. This extends the work done in Ref. [22] on electron beam deflection. PFT is the first-order spatiotemporal coupling of the laser pulse envelope (higher-order terms, including pulse front curvature, are not in the scope of the present work). We hereafter derive an expression for laser deflection in a plasma and validate it using two-dimensional (2D) particle-in-cell (PIC) simulations. We also incorporate laser deflection into the model of electron steering due to PFT developed in Ref. [22] and develop a complimentary deflection model due to transverse plasma density gradients. In the last section, we present experimental results obtained on the BELLA (BErkeley Laboratory Laser Accelerator) petawatt system at the BELLA Center at the Lawrence Berkeley National Laboratory [23]. Both laser and electron steering show clear dependence on angular dispersion and group delay dispersion, leading to mrad-order deflection. We are able to controllably steer the electron beam by adjusting the laser parameters without significant degradation of electron beam properties. Previous experimental work [18] on PFT steering explored only near-field angular dispersion, whereas this work includes the effect of temporal dispersion [24], which is qualitatively different.

\section{LASER STEERING}

A transverse gradient in refractive index results in deflection of a propagating laser pulse. The local deflection angle $\theta \ll \pi / 2$ of light is related to the transverse gradient in the 
index of refraction by [25]

$$
\frac{d \theta_{l}}{d z}=\frac{1}{\eta} \frac{\partial \eta}{\partial x}
$$

where $\eta$ is the plasma index of refraction, $x$ is a transverse coordinate, and $z$ is the laser propagation direction. In an LPA, this transverse gradient can result from density gradients in the plasma target, or it can arise due to asymmetries in the laser pulse.

For low-intensity laser pulses (where the electron quiver motion is nonrelativistic), the index of refraction of the plasma reads $\eta=\left(1-n_{e} / n_{c}\right)^{1 / 2}$ where $n_{e}$ is the local electron density and $n_{c}$ is the plasma critical density for the laser angular frequency $\omega_{L}$, defined by $n_{c}=m_{e} \epsilon_{0} \omega_{L}^{2} / e^{2}$ where $\epsilon_{0}$ is the vacuum permittivity, $m_{e}$ is the mass of an electron, and $e$ is the elementary charge. The plasma angular frequency is $\omega_{p}=$ $\omega_{L}\left(n_{0} / n_{c}\right)^{1 / 2}$ and its wave number is $k_{p}=\omega_{p} / c$. A linear transverse gradient in the plasma density with characteristic length $L_{t}$, i.e., $n_{e}=n_{0} \times\left(1+x / L_{t}\right)$, where $n_{0}$ is the plasma density on-axis, results in deflection or the laser pulse [21]. For a low-density plasma $\left(n_{e} \ll n_{c}\right)$, the laser deflection is given by

$$
\frac{d \theta_{l}}{d z} \simeq n_{0} /\left(2 n_{c} L_{t}\right)
$$

When a high-intensity laser pulse propagates in plasma, it generates a plasma wave (the laser wake) that affects its propagation. As the plasma density varies within the laser pulse, different regions of the pulse experience different values of the index of refraction. This well-known effect is at play in self-focusing [26]. Laser steering from a tilted pulse arises from the same effect, and in this section we derive the average steering rate of a tilted laser pulse as a function of laser and plasma parameters.

We hereafter consider a linearly polarized laser pulse propagating along $z$, transversely and temporally Gaussian, with duration $\tau$, spatial size $w=w(z)\left(1 / e^{2}\right.$-intensity radius), wavelength $\lambda_{L}$, angular frequency $\omega_{L}$, and wave number $k_{L}$. The pulse length is $L=c \tau$ and the normalized laser amplitude at focus is $a=e A /\left(m_{e} c\right)=\bar{a} \cos \left(k_{L} \xi\right)$, where $c$ is the speed of light, $A$ is the laser vector potential, $\xi=z-c t$ is the comoving coordinate, and the overbar stands for the slowly varying envelope. Pulse front tilt (PFT) occurs when the laser pulse front, which is based on intensity, is not parallel to the wavefronts, i.e., the laser pulse appears to be tilted with respect to its propagation direction, as illustrated in the bottomright diagram in Fig. 1. The envelope of the normalized vector potential $\bar{a}$ in the presence of PFT is given by

$$
\bar{a}(\xi, x)=a_{0} e^{-(\xi+x \tan \psi)^{2} / L^{2}} e^{-x^{2} / w^{2}},
$$

where $\psi$ is the pulse front tilt angle and $a_{0}$ is the peak normalized vector potential. In what follows, PFT is introduced in the $x$ direction, without loss of generality.

As described in Ref. [24], PFT results from the combination of (1) group delay dispersion $\varphi^{(2)},(2)$ spatial dispersion $\zeta$, and (3) angular dispersion $\beta$. These parameters are illustrated in Fig. 1 and defined in Ref. [24]. The vacuum PFT angle $\psi$ a distance $z$ from focus of a paraxial $\left(k_{L} w_{0}>1\right)$ laser pulse that is Gaussian in space and time (and spectrum) is given by

$$
\tan \psi=\frac{c k_{L}^{2} w_{0}^{2}\left[\beta_{0} k_{L} \tau_{0}^{2} w_{0}^{2}+4 \zeta_{0}\left(\varphi_{0}^{(2)}+\beta_{0} k_{L} \zeta_{0}\right)\right]-4 c k_{L}\left[\tau_{0}^{2} \zeta_{0}-\beta_{0} k_{L} w_{0}^{2}\left(\varphi_{0}^{(2)}+\beta_{0} k_{L} \zeta_{0}\right)\right] z}{k_{L}^{2} w_{0}^{2}\left(\tau_{0}^{2} w_{0}^{2}+4 \zeta_{0}^{2}\right)+\left(8 \beta_{0} k_{L}^{2} w_{0}^{2} \zeta_{0}\right) z+4\left(\tau_{0}^{2}+\beta_{0}^{2} k_{L}^{2} w_{0}^{2}\right) z^{2}}
$$

where $\tau_{0}$ is the transform-limited pulse length (i.e., without PFT or $\varphi_{0}^{(2)}$, defined as the $1 / e^{2}$-intensity half-width), $w_{0}$ is the focal spot size without PFT $\left(1 / e^{2}\right.$-intensity radius), and the quantities $\beta_{0}, \zeta_{0}$, and $\varphi_{0}^{(2)}$ are specified at focus. These far-field quantities are related to the near-field values $\beta, \zeta$, and $\varphi^{(2)}$ by

$$
\beta_{0}=-\zeta / f, \quad \zeta_{0}=\beta f, \quad \varphi_{0}^{(2)}=\varphi^{(2)}+2 k_{L} \beta \zeta,
$$

which are defined a distance $1 f$ before the focusing optic with focal length $f$ (13.5 $\mathrm{m}$ for BELLA). The first two of these can be understood by considering the action of a focusing optic, which converts near-field spatial position $(\zeta \omega)$ to angle at focus $\left(\beta_{0} \omega\right)$ and vice versa. The third is the increase in $\varphi^{(2)}$ with propagation due to the presence of $\beta$, with an additional term that arises from cross-coupling between spatial and angular dispersion at the focusing optic. Note that the pulse length $\tau$ changes as the laser propagates due to the changing value of $\varphi^{(2)}$. For example, a Gaussian pulse with transform-limited duration $\tau_{0}=30 \mathrm{fs}$ ( $35 \mathrm{fs}$ full width at half maximum intensity) doubles in length from $\varphi^{(2)}=780 \mathrm{fs}^{2}$.

In Eq. (4) the laser beam is not assumed to be collimated. Hence, the conversion between angular chirp in the near field and spatial chirp in the far field is taken into account, which explains the slight difference between this equation and Eq. (33) in Akturk et al. [24], which does assume collimation.

The plasma index of refraction and density are functions of the normalized pseudopotential, defined as $\Psi=e V /\left(m_{e} c^{2}\right)-$ $a_{z}$ where $V$ is the electrostatic potential and $a_{z}$ is the longitudinal component of the vector potential. The pseudopotential behind the laser pulse can be written in a closed form provided that the laser-plasma interaction is in the linear regime, where the laser spot size is sufficiently large $\left(k_{p} w \gtrsim 1\right)$ and the laser pulse is not too intense $\left(a_{0} \lesssim 1\right)$. In the linear regime, the pseudopotential is given by [27]

$$
\Psi(\xi, x)=\int_{\xi}^{\infty}-\frac{1}{4} \bar{a}^{2}(\xi, x) \sin \left[k_{p}\left(\xi-\xi^{\prime}\right)\right] k_{p} d \xi^{\prime} .
$$

For a Gaussian pulse, the closed-form solution to Eq. (6) is given by

$$
\begin{aligned}
\Psi(\xi, x)= & \frac{-k_{p} L}{8} a_{0}^{2} \sqrt{\frac{\pi}{2}} e^{-\left(k_{p} L\right)^{2} / 8} e^{-2\left(x^{2}+y^{2}\right) / w^{2}} \\
& \times \operatorname{Im}\left\{e^{i k_{p} \xi}\left[1-\operatorname{Erf}\left(\frac{\sqrt{2}}{L} \xi+\frac{i k_{p} L}{2 \sqrt{2}}\right)\right]\right\} .
\end{aligned}
$$




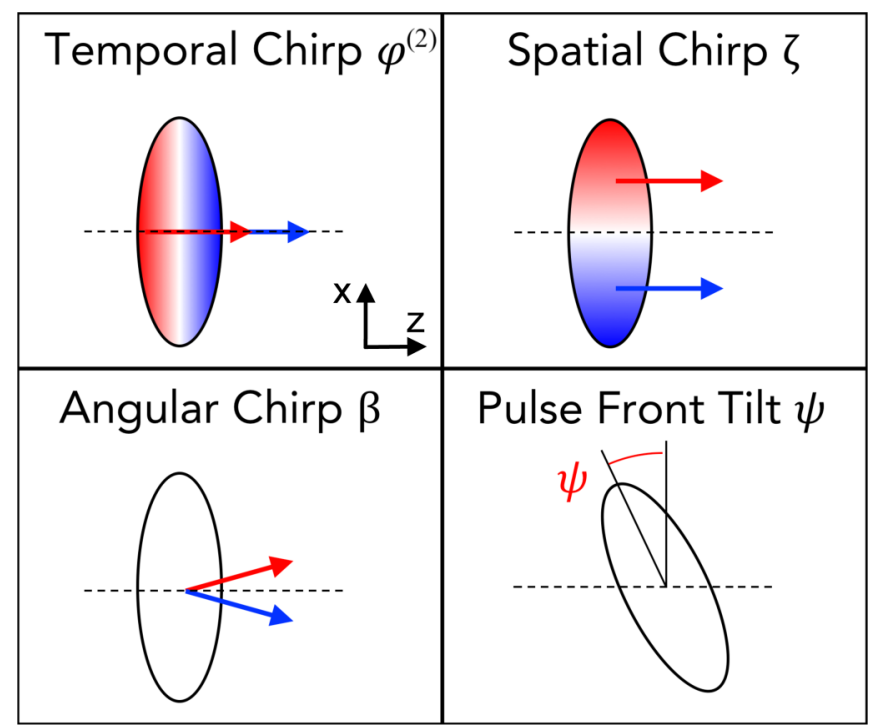

FIG. 1. Illustration of parameters relevant for this study. Pulse front tilt (PFT) from temporal chirp, spatial chirp, and angular dispersion is given by Eq. (4). The dashed line shows the propagation axis along which the laser pulse propagates to the right. Colors indicate different spectral components.

In this regime, $\Psi$ is calculated through integration along the $z$ axis [27], so the result with PFT can be readily derived by making the substitution $\xi \rightarrow \xi+x \tan \psi$.

The index of refraction is related to $\Psi$ by

$$
\eta=\sqrt{1-\frac{\bar{n}_{e}}{\gamma n_{c}}} \approx 1-\frac{n_{0}}{2 \gamma n_{c}}\left(1+\frac{1}{k_{p}^{2}} \nabla^{2} \Psi\right),
$$

where $\gamma=\sqrt{1+\bar{a}^{2} / 2}$ is the relativistic factor acquired by electrons due to the oscillations in the laser field, and we assumed $\bar{n}_{e} \ll n_{c}$. The spatially dependent electron density $\bar{n}_{e}$ is averaged over one laser period, to remove the fast oscillations at $\omega_{L}$. We hereafter calculate the average steering of the laser pulse.

Note that Eqs. (6) and (8) depend only on $\bar{a}$ and not on the fast oscillations of the laser pulse, since the plasma reacts on a $k_{p}$-length scale $\left(k_{p}<<k_{L}\right)$. For this reason, the laser steering is independent of the polarization direction of the laser pulse. This justifies the earlier statement that PFT can be introduced in the $x$ direction without loss of generality.

The deflection of a Gaussian pulse with length $L \lesssim \lambda_{p} / 2$ can be calculated by substituting Eq. (7) into Eq. (8) with the substitution $\xi \rightarrow \xi+x \tan \psi$, taking the transverse derivative indicated in Eq. (1), and calculating the intensity-weighted average. The limitation on pulse length arises from averaging Eq. (1) over the pulse. For pulses with $L>\lambda_{p} / 2$, the head of the pulse experiences a different gradient from the tail, which can seed hosing [20].

The calculation can be considerably simplified with two approximations. The first is to drop the factor $1 / \eta \approx 1$ in Eq. (1), which is valid in the underdense linear regime where $a_{0} \ll 1$ and $n_{0} \ll n_{c}$. The second is to replace $\gamma$ with its peak value $\gamma_{0}$ in Eq. (8) so that the only remaining $x$ dependence is in the $\Psi$ term. This is possible because of the subsequent intensity-weighted average, which limits the influence of the

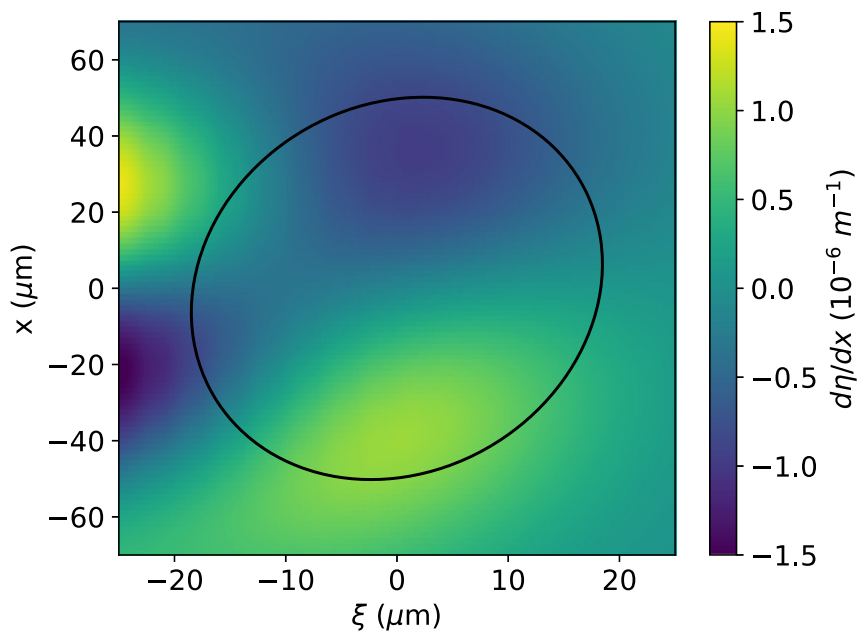

FIG. 2. Laser-induced gradient of refractive index in a 2D LPA simulation with a $60 \mathrm{fs}$ laser pulse propagating along $z$ with $a_{0}=$ 0.01 focused to a $w_{0}=50 \mu \mathrm{m}$ waist in a uniform plasma with initial density $10^{16} \mathrm{~cm}^{-3}\left(\lambda_{p}=330 \mu \mathrm{m}\right)$. PFT is due to $\zeta_{0}=1 \mathrm{~mm} \mathrm{fs}$ and $\varphi_{0}^{(2)}=-500 \mathrm{fs}^{2}$ at focus $(\psi=-46 \mathrm{mrad})$. The black solid-line ellipse is an isocontour of laser intensity where the intensity is half of the peak intensity.

pulse volume where $\gamma$ is significantly lower than its peak value. The resulting expression for laser deflection rate in the plane of pulse front tilt is

$$
\begin{aligned}
\left\langle\frac{d \theta_{l}}{d z}\right\rangle= & \frac{-a_{0}^{2}}{\gamma_{0}} \frac{n_{0}}{n_{c}}\left(k_{p} \tan \psi\right)\left(k_{p} L\right) e^{-\left(k_{p} L\right)^{2} / 4} \\
& \times \frac{\sqrt{\pi}}{32} \times \begin{cases}1 & \text { (2D) } \\
\frac{1}{\sqrt{2}} & \text { (3D). }\end{cases}
\end{aligned}
$$

Equation (9) can be understood qualitatively by examining the index of refraction $\eta$ for a tilted pulse front. A transverse gradient in $\eta$ (plotted in Fig. 2) arises because the laser pulse has finite size, so the wake amplitude decreases as $|x| \rightarrow$ $\infty$. This transverse gradient is responsible for self-focusing, although here $k_{p} L \lesssim 1$ so the effect is to slightly defocus the laser pulse. Due to the asymmetry of the wake caused by the PFT, the transverse gradient of the index of refraction is directed upward (positive) on average in the pulse region. The steering rate in Eq. (9) scales as $a_{0}^{2} / \gamma_{0}$, which is the scaling of the wake amplitude [27] and is linearly proportional to the PFT $\psi$ when $\psi \ll \pi / 2$. Factors of $k_{p} L$ give the coupling between laser pulse length and plasma wavelength, which is optimal when $k_{p} L \simeq 1$.

Two-dimensional PIC simulations were performed with the WARP code $[28,29]$ in a boosted frame [30] to test the predictions of Eq. (9). The laser deflection observed in PIC simulations is shown in Figs. 3(a)-3(d) as a function of the main parameters and compared with the predictions of Eq. (9). In Fig. 3(a) the variation of laser pointing deflection rate as a function of PFT angle $\psi$ is shown to be linear for small angles. All predictions are in good agreement with PIC simulations. The derivation of Eq. (9) assumed $a_{0} \ll 1$, since Eq. (6) is valid only in this regime, but the theoretical value does not diverge from the deflection observed in PIC simulation until $a_{0} \approx 2$. The dependence on pulse length and density is 
(a)

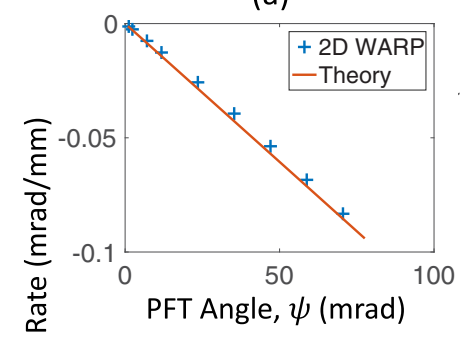

(c)

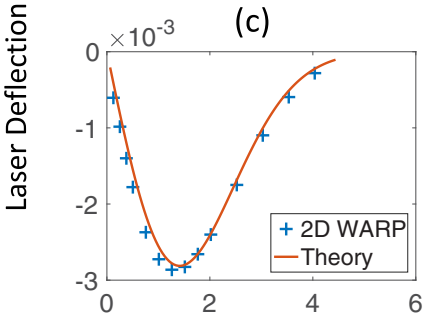

Normalized Pulse Length, $k_{p} L$ (b)

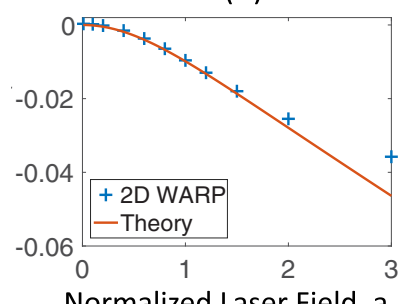

(d)

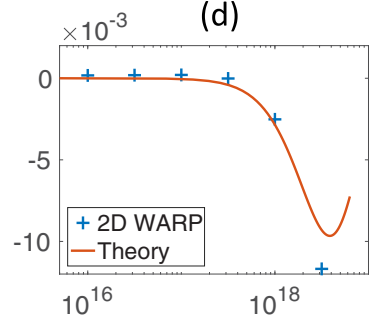

Electron Density, $\mathrm{n}_{0}\left(\mathrm{~cm}^{-3}\right)$

FIG. 3. Laser steering rate from 2D PIC simulations with WARP (blue crosses) and theory (red line) as a function of main physical parameters. Unless specified otherwise, nominal parameters are as follows: the background plasma density is $n_{0}=1 \times 10^{18} \mathrm{~cm}^{-3}$, the laser normalized amplitude is $a_{0}=0.5$, the waist is $w_{0}=150 \mu \mathrm{m}$ so that the pulse remains roughly collimated during the $5 \mathrm{~mm}$ propagation $\left(z_{R} \simeq 90 \mathrm{~mm}\right)$, and PFT around focus is introduced with $\beta=5$ as at focus (i.e., in the far field) with $\lambda=800 \mathrm{~nm}$. The pulse duration is $\tau_{0}=30 \mathrm{fs}$ without PFT. The steering rate $\left\langle d \theta_{e} / d z\right\rangle$ is obtained by averaging $d^{2} x_{c} /\left(c^{2} d t^{2}\right)$ over the $5 \mathrm{~mm}$ propagation where $x_{c}$ is the transverse position of the laser pulse centroid. Subplots show the steering rate as a function of the following parameters: (a) PFT angle, (b) laser field amplitude, (c) pulse duration (for this scan, the parameters were slightly different, with $a_{0}=0.8$ and $n_{0}=2 \times$ $10^{17} \mathrm{~cm}^{-3}$ ), and (d) plasma density.

shown in Figs. 3(c) and 3(d), respectively. The discrepancy for the highest density in Fig. 3(d) is due to the onset of self-focusing, which modifies the laser properties, including PFT, and thus changes the deflection rate. These plots show excellent agreement between the model of Eq. (9) and the PIC simulation results in the linear and quasilinear regimes $\left(a_{0} \leqslant 2\right)$, provided the density is low enough to avoid strong self-focusing. We speculate that our theory works for $a_{0}>1$ because short pulses $\left(L \lesssim \lambda_{p} / 2\right)$ interact only with the front of the plasma wave, which does not become nonlinear with increasing $a_{0}$ as quickly as the rest of the wake.

\section{ELECTRON BEAM STEERING}

The laser wake provides both accelerating and focusing forces on the electron beam. In an axisymmetric wake, electrons perform betatron oscillations around the $z$ axis where the transverse focusing fields vanish, $F_{\perp}(x=0, \xi)=0$. If, however, the wake is asymmetric (e.g., due to PFT), the condition $F_{\perp}\left(x=x_{E q}, \xi\right)=0$ is met off-axis. This causes an electron beam located $\xi_{e}$ behind the laser to perform betatron oscillations around an off-axis transverse equilibrium position $x_{E q}=x_{E q}\left(\xi_{e}\right)$. This effect is described in Ref. [22] and briefly summarized here. The evolution of the instantaneous electron beam transverse position $x_{e}$ near the equilibrium position $x_{E q}$

is described by

$$
\frac{d^{2} x_{e}}{d t^{2}}+c^{2} k_{\beta}^{2}\left(x_{e}-x_{E q}\right)=0
$$

where $k_{\beta}$ is the betatron wave number. The location of the equilibrium position $x_{E q}$ at a particular $\xi$ in the linear regime is the solution to

$$
\tan \psi+\frac{4 x_{E q}}{k_{p} w^{2}} \tan \left[k_{p}\left(\xi-x_{E q} \tan \psi\right)\right]=0 .
$$

If the laser is also deflecting (see previous section), $x$ should be replaced with $x-x_{L}(z)$ where $x_{L}(z)$ is the laser transverse position. The equilibrium position $x_{E q}$ can evolve due to changes in the plasma $\left(k_{p}\right)$, the laser $(w$ and $\psi$ ), or the dephasing of the electrons since $x_{E q}=x_{E q}(\xi)$ (typically less significant since the electron changes phase slowly compared to the plasma density, e.g., in a gas cell). When the equilibrium position $x_{E q}$ evolves during propagation, the average electron beam position moves transversely, leading to electron deflection.

A transverse density gradient also distorts the laser wake, which in turn affects the electron beam direction. As done in Ref. [22], we hereafter calculate the positions where the transverse force is zero $\left(F_{\perp}=0\right)$ in the presence of a linear transverse density gradient $L_{t}$ in the $x$ direction, i.e., $n(x, z)=n(z) \times\left(1+x / L_{t}\right)$. Assuming the transverse gradient length is much larger than the beam width $w, L_{t} \gg w$, the pseudopotential behind the laser is $\Psi=\Psi_{0} \sin \left\{k_{p} \xi[1+\right.$ $\left.\left.x /\left(2 L_{t}\right)\right]\right\} \exp \left(-2 x^{2} / w^{2}\right)$, where $\Psi_{0}$ is a constant depending on the pulse intensity and shape and $k_{p}$ is the initial on-axis plasma wave number $\left(x=0\right.$ and $\xi \gg 0$ ). Solving for $F_{x}=0$ (i.e., $\partial_{x} \Psi=0$ ) gives the equation for the $x-\xi$ position where the transverse force is zero as

$$
\frac{8 x L_{t}}{w^{2}}\left(1+\frac{x}{L_{t}}\right) \frac{\tan \left[k_{p} \xi\left(1+\frac{x}{2 L_{t}}\right)\right]}{k_{p} \xi\left(1+\frac{x}{2 L_{t}}\right)}=1 .
$$

Again, the solution $x_{E q}$ varies with position $\xi$.

Figure 4 shows a snapshot of the transverse electric field from a PIC simulation of laser wakefield acceleration in the linear regime where (a) the plasma density has a linear transverse gradient (no PFT) and (b) the laser has PFT (uniform density). The red line shows $E_{x}=0$ from the simulation, which is not along the $z$ axis. The black dashed line is the numerical solution of Eq. (12) in Fig. 4(a) and Eq. (11) in Fig. 4(b). Both show excellent agreement with the PIC simulation. As can be seen on this image, a transverse gradient in the plasma density distorts the plasma wake in a very similar way as laser PFT. Hence, the conclusions of Ref. [22] also apply in the presence of a transverse density gradient, and we refer to this article for further details. Note that in the case of PFT, the wake distortion is periodic (the distortion is the same in the first, second, and third bucket) whereas, in the presence of a transverse gradient, the wake distortion increases with the distance to the laser pulse (e.g., the second bucket shows stronger distortion than the first). This is because the wake accumulates in the transverse phase with increasing distance behind the laser pulse, due to the difference in plasma period across the wake. In contrast, the transverse wake phase added by the tilted laser pulse (PFT) is the same for all buckets. This effect might impose additional constraints to inject electron beams in multiple buckets. 


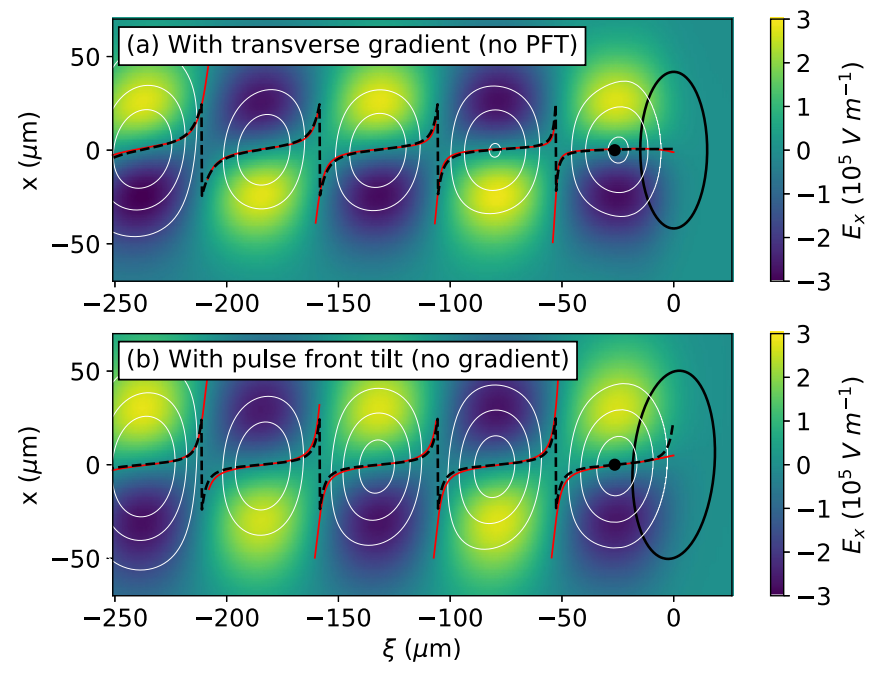

FIG. 4. (a) Transverse electric field $E_{x}$ for a $y$-polarized Gaussian laser pulse with $a_{0}=0.01, w_{0}=50 \mu \mathrm{m}, \tau_{0}=60 \mathrm{fs}$, and focused at $z=100 \mu \mathrm{m}$. The laser has no PFT. The plasma density is $n_{e}=10^{17} \mathrm{~cm}^{-3}$ with a transverse linear gradient with characteristic length $L_{t}=1 \mathrm{~mm}$. The $2 \mathrm{D} x-z$ simulation box is $300 \mu \mathrm{m} \times 300 \mu \mathrm{m}$ with $256 \times 4096$ grid points. The simulation was performed with the PIC code WARPX [31] in a Lorentz boosted frame [30]. Black ellipse: isocontour of the laser pulse envelope. Red line: $E_{x}=0$ from the PIC simulation. Black dashed line: model for $E_{x}=0$. White lines: isocontours of the pseudopotential $\Psi$. (b) Same quantities in the presence of PFT due to $\varphi_{0}^{(2)}=-500 \mathrm{fs}^{2}$ and $\zeta_{0}=1 \mathrm{~mm}$ fs, with no transverse density gradient. Note that in (b) the line where $E_{x}=0$ (black dashed line) is the same in each bucket, whereas in (a) the angle of the line increases with subsequent buckets behind the laser pulse.

Wake deformation due to PFT and/or transverse density gradient shifts the equilibrium position for the electron beam in an LPA to $x_{E q} \neq 0$. Electron steering occurs when $x_{E q}$ evolves along the propagation, either from laser evolution or longitudinal variation of the plasma density profile. In particular, changes in laser or plasma parameters that cause sharp changes in $x_{E q}$ can seed coherent betatron oscillations [see Eq. (10)]. Even if the PFT and density do not change with propagation, the electron beam can steer off axis due to laser deflection. For a finite-length LPA, the electron beam can also deflect due to the longitudinal density downramp at the end of the plasma target, where $k_{p}$ and $k_{\beta}$ change rapidly leading to electron beam deflection relative to the laser. As demonstrated in Ref. [22], the deflection depends strongly on the longitudinal density gradient as well as on the sign of the PFT and/or transverse gradient. Experiments illustrating these effects are presented below.

\section{EXPERIMENTS}

\section{A. Setup and diagnostics}

Experiments were performed at the BErkeley Laboratory Laser Accelerator (BELLA) Center to measure the dependence of laser deflection and electron pointing on pulse front tilt (PFT). The laser plasma accelerator consisted of the BELLA petawatt laser, operated at a peak power of $300 \mathrm{TW}$, and a supersonic helium gas jet target. The laser had a central wavelength on $\lambda_{L}=810 \mathrm{~nm}$ and a focal spot in vacuum of $52 \mu \mathrm{m}$ full width at half maximum (FWHM). Simulations [32-34] show that the laser self-focuses to a peak value of $a_{0} \approx 3$ in the plasma, so the experimental LPA is in the nonlinear regime and electrons are injected by wave breaking of the laser wake.

The final optical compressor was adjusted to add near-field angular dispersion and group delay dispersion to the laser, which together produce PFT at focus [see Eq. (4)]. The compressor is located $\sim 1 f$ before the final focusing optic, so the near-field values $\beta, \zeta$, and $\varphi^{(2)}$ are defined at the compressor. The laser polarization at the compressor and at the plasma was horizontal, but there is no polarization dependence in the theory developed above. Angular dispersion was added to the laser in the vertical direction via the rotation of the final grating of the four-grating compressor. The rotation was about the grating surface normal, rather than in the dispersion plane, so the gratings' faces remain parallel, which minimizes the high-order spectral phase terms caused by nonparallel or unmatched gratings [35]. The angular dispersion as a function of grating rotation was measured using a GRENOUILLE [36]. A rotation of $10 \mu \mathrm{rad}$ corresponds to $\beta \approx-10 \mathrm{zs}$. However, the zero point of PFT depends on the GRENOUILLE alignment, so a second diagnostic (focal spot size) was used to determine the grating rotation corresponding to $\beta=0 \mathrm{zs}$. The reproducibility of the translation stage for grating rotation as well as the uncertainty in the minimum location gives a $\beta$ uncertainty of $\sim 5 \mathrm{zs}$. There is only a $6 \%$ reduction in peak fluence at vacuum focus (corresponding to a $\sim 6 \%$ change in vertical focal spot size) for $\beta=20 \mathrm{zs}$, the largest angular dispersion used in experiments. The GRENOUILLE measurements show the contribution of near-field $\zeta$ to the PFT at focus was minimal, so we assume $\zeta=0$ for the simulations and experiments that follow.

The added angular dispersion is related to the grating rotation by [37]

$$
\beta=\frac{-\lambda^{2}}{2 \pi c} G \delta,
$$

where $\delta$ is the rotation angle and $G$ is the grating groove density (1480 lines $/ \mathrm{mm}$ ). The small resulting pointing change of the laser was compensated after each adjustment using the mirror immediately following the optical compressor. This ensured that the alignment of the laser remained fixed in both the near and far fields. The PFT which arises from the added angular dispersion [see Eq. (4)] was controlled by adjusting the grating spacing, which changes the $\varphi^{(2)}$ of the pulse and does not affect laser alignment. (An added $\varphi^{(2)}= \pm 500 \mathrm{fs}^{2}$ results in a $\sim 20 \%$ drop in laser peak power.) The deflection of the laser and electron beams resulting from added PFT was then recorded as a function of $\varphi^{(2)}$ for several values of $\beta$.

The plasma target was a pulsed supersonic gas jet with a $15 \mathrm{~mm}$ slit geometry operated with helium gas. The threedimensional density profile of the gas jet was characterized by neutral gas tomography [38,39], in a separate set of measurements from the LPA experiment. To verify the absolute density in the experiment, the laser spectral shift resulting from the laser-plasma interaction was compared to the spectral shift from simulations of laser evolution performed using the INF\&RNO code [32-34], similarly to Ref. [3]. The best 


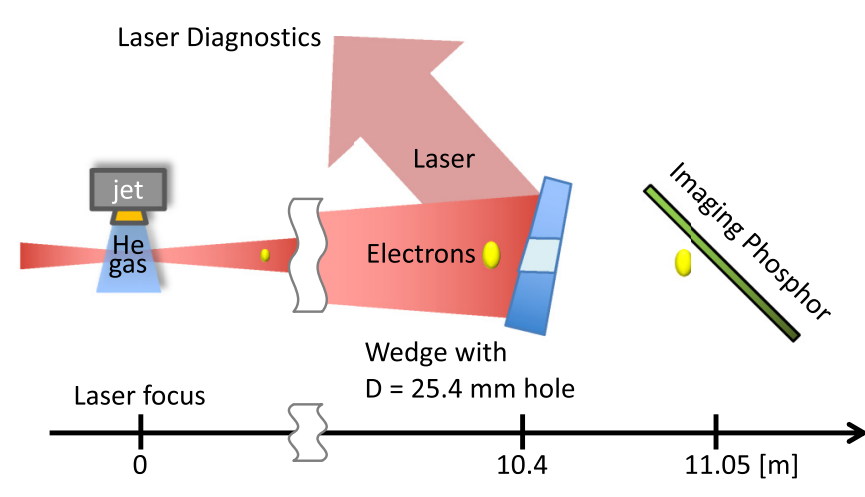

FIG. 5. The BELLA experimental setup for these experiments. Accelerated electrons were separated from the postinteraction laser pulse by optics with \pm 1.2 mrad clearance. The first optic after the interaction (wedge with hole) was imaged to determine laser deflection. An imaging phosphor was used to determine electron deflection.

agreement between INF\&RNO simulations and measurements was obtained when the tomographic density profile was scaled to a peak density of $8.7 \times 10^{17} \mathrm{~cm}^{-3}$ (scaling factor 0.83 ). The discrepancy in retrieved density between the tomographic and simulation methods is due to several factors, including time dependence in the gas density that varies with duty cycle and operation time (due to thermal effects in the valve solenoid). For further details, see Ref. [40].

The laser deflection was measured by imaging the nearfield laser mode on the first optic after the plasma target, an uncoated wedged glass flat with a $25.4 \mathrm{~mm}$ diameter hole (see Fig. 5). Aberrations in the imaging system were corrected for in the analysis. The hole in the optic was numerically filled in the images, but the pointing analysis was found to be insensitive to the interpolation technique utilized since the hole was smaller than the near-field mode size. The laser direction was then taken as the centroid of the corrected intensity distribution, after applying a threshold to remove stray light. The effect of the intensity threshold is discussed in the Appendix. The zero point of laser deflection is the laser direction in vacuum.

The electron pointing direction was determined from the insertable phosphor screen, which yielded the angular distribution of charge density. The standard deviation of electron pointing from shot-to-shot jitter was $\sim 0.2 \mathrm{mrad}$, which allowed the investigation of sub-mrad steering from PFT effects (laser pointing stability is $<5 \mu \mathrm{rad}$ [23]). However, the electron beams were partially clipped when the beams were significantly deflected (see Fig. 6) because the divergence of the electron beams ( $\sim 1 \mathrm{mrad})$ was close to the angular acceptance of the diagnostic $( \pm 1.2 \mathrm{mrad})$, set by the hole in the laser optics after the target (see Fig. 5). The pointing direction was taken as the geometrical center of the elliptical distribution, which was determined by an elliptical fit to the contour in the charge density distribution at $70 \%$ of the peak charge density. Results were insensitive to $\pm 20 \%$ changes in charge density threshold. A second method of determining the electron beam pointing, the centroid of the electron distribution on the phosphor, agrees with the elliptical fit method over the offset range where the electron beam is not significantly clipped. The fit method was possible because the electron

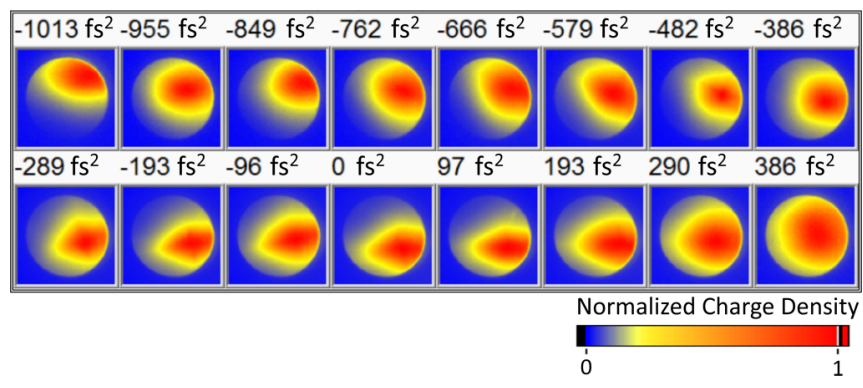

FIG. 6. Plots of electron charge distribution (normalized to unity) measured by the insertable phosphor. The laser group delay dispersion (shown above each plot) was varied with fixed near field $\beta=9.7 \mathrm{zs}$, resulting in electron deflection due to pulse front tilt. The radius of the circle is $1.2 \mathrm{mrad}$, set by the hole in the upstream laser optics. The electron beams maintain a consistently elliptical shape, which allows the use of contour fitting for electron pointing determination [provided less than $50 \%$ of the charge (ellipse) area is clipped].

beam distributions were consistently elliptical, as shown in Fig. 6. The electron beam deflection on the phosphor shown in Fig. 6 is a nonlinear function of $\varphi^{(2)}$ which modifies the PFT. The electron beam starts high, moves downward, and then moves upward after $\varphi^{(2)}$ changes sign (see Fig. 9 below for a plot of vertical position).

\section{B. Results}

The model for laser steering presented in Sec. II relies on the knowledge of pulse front tilt (PFT) along propagation. While this can be calculated analytically for a Gaussian pulse with angular, temporal, and/or spatial chirp propagating in vacuum, the PFT in a plasma is modified by evolution of the laser pulse, such as self-focusing, and can be affected by laser imperfections. The PFT evolution in a plasma can differ very strongly from its evolution in vacuum, and to our knowledge there is no predictive model to describe the PFT evolution in a plasma in the nonlinear regime. As an illustration, Fig. 7 shows the PFT evolution for a Gaussian laser pulse in vacuum and in a plasma in the nonlinear regime comparable with the experimental conditions. Since the laser PFT along propagation in the plasma is not known in our

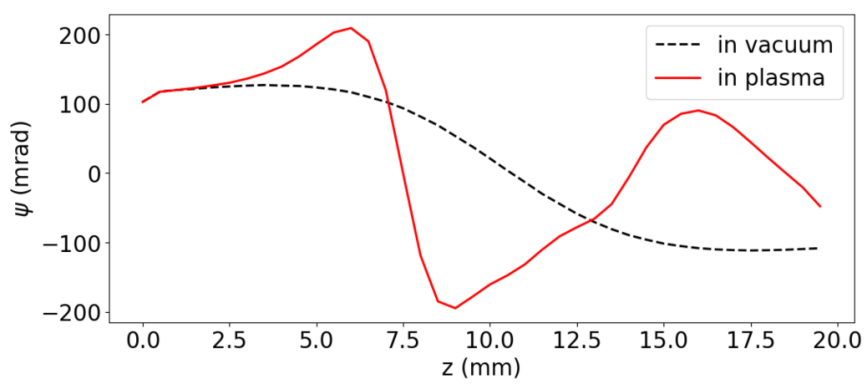

FIG. 7. Evolution of the PFT of a laser pulse along propagation in vacuum (black dashed line) and in a uniform plasma (red solid line). The plasma density is $n_{0}=10^{18} \mathrm{~cm}^{-3}$, and the Gaussian laser pulse is focused at $z=10 \mathrm{~mm}$ with $a_{0}=3, w_{0}=35 \mu \mathrm{m}$ and duration $\tau_{0}=40 \mathrm{fs}$. PFT is due to $\zeta_{0}=0.7 \mathrm{~mm}$ fs at focus with $\varphi_{0}^{(2)}=\beta_{0}=0$ [see Eq. (4)]. These 3D simulations were performed with WARPX in a boosted frame with $\gamma_{\text {boost }}=15$. 
(a) Measured Laser Angle

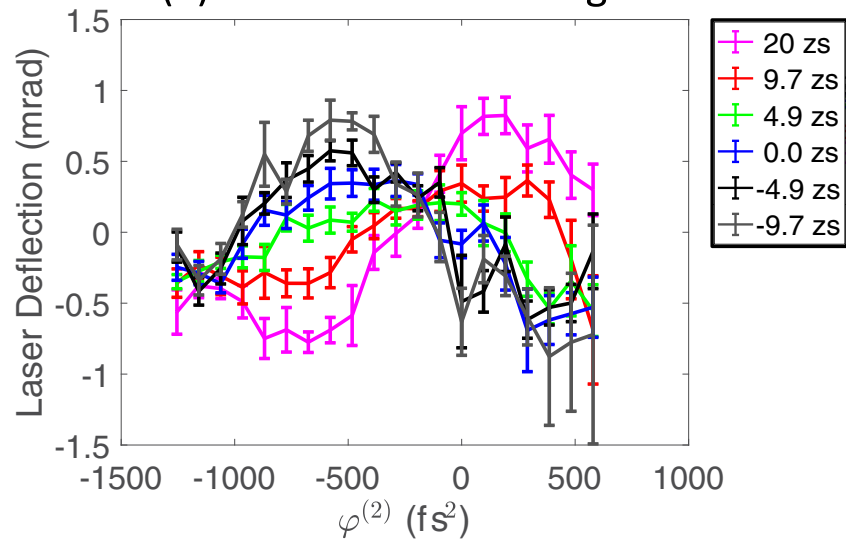

(b) Calculated Laser Angle

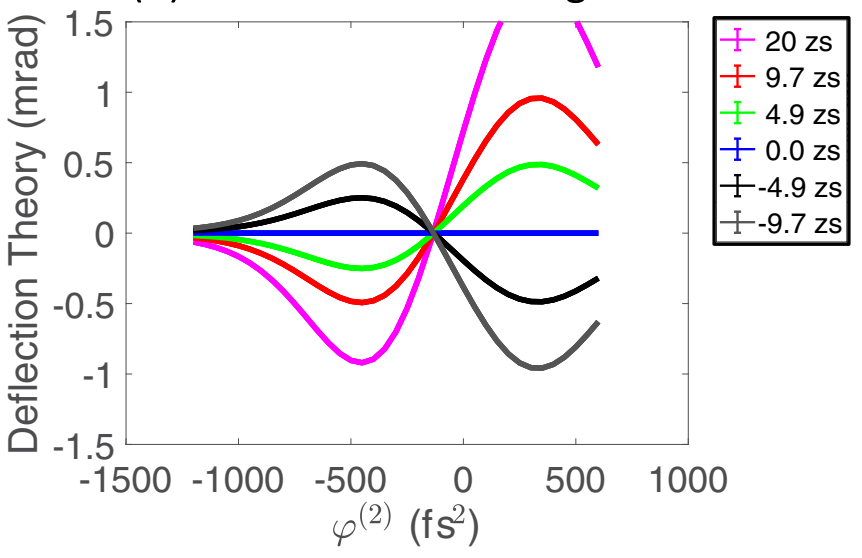

FIG. 8. Experimental results and comparison with theory. (a) Final laser angle in the experiment as a function of group delay dispersion $\varphi^{(2)}$ for several values of near-field angular dispersion $\beta$. (b) Laser deflection calculated from the model for the same parameters as the experiment. The nonzero $\varphi^{(2)}$ crossing point and deflection amplitude agree between experiment and theory.

experiment, we modeled the experimental results with two simplifying assumptions: (1) the laser was Gaussian in time and space and (2) the laser propagation was not affected by the plasma (i.e., the intensity and PFT evolve as in vacuum). Although these assumptions [in particular (2)] do not hold for the experimental conditions, they give a simple estimate of the laser deflection that does not require computationally intensive PIC simulations. With these assumptions, the laser steering model can be applied, and the final laser direction is obtained by integrating Eq. (9) along the plasma profile.

The measured laser deflection as a function of $\varphi^{(2)}$ is plotted in Fig. 8(a). Each curve corresponds to a fixed value of $\beta$ [see Eq. (13)]. The amplitude of the laser deflection is proportional to the added angular dispersion, which is expected since PFT at focus scales linearly with added angular dispersion. However, the laser deflection is not linear with $\varphi^{(2)}$ due to the dependence of pulse length, peak intensity, and PFT on $\varphi^{(2)}$. The results in Fig. 8(a) are sensitive to the intensity threshold used in the pointing analysis, which adds systematic uncertainty in addition to the statistical uncertainty indicated by the error bars (95\% confidence level in the mean)

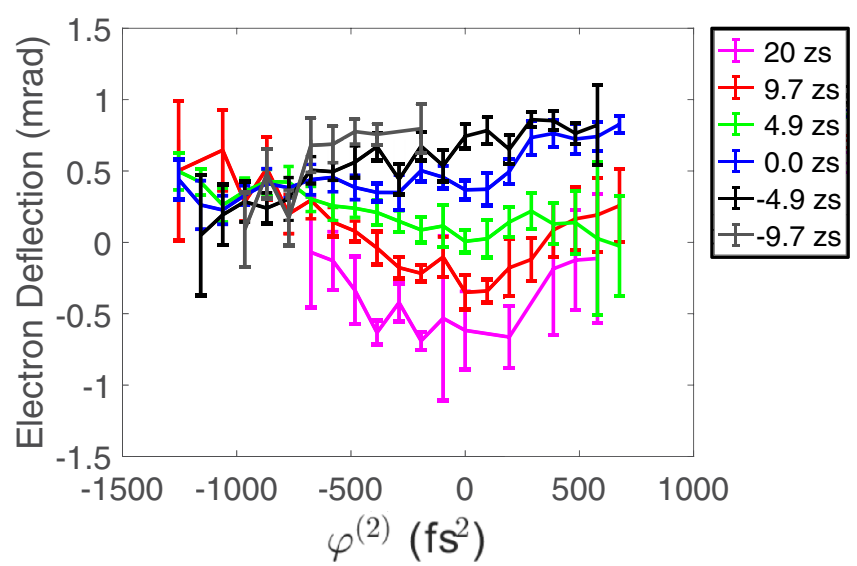

FIG. 9. Measured electron pointing as a function of $\varphi^{(2)}$ for several values of $\beta$. The electron beam direction differs from the laser in Fig. 8, indicating a distinct mechanism is deflecting the electrons.

from shot-to-shot fluctuations. The effect of this threshold was investigated [40], and the crossing point and order of the plots in Fig. 8(a) do not change significantly with threshold. (See the Appendix for a plot illustrating the effect of intensity threshold on pointing determination for a wide range of threshold values.)

Figure 8(b) shows the prediction of the model [i.e., integration of Eq. (9)] for the six values of angular dispersion used in the experiment and shown in Fig. 8(a). In spite of the simplified assumptions, the model predictions are qualitatively consistent with experimental results. In particular, the model correctly predicts the crossing point as well as the amplitude of the laser deflection. The plots of deflection cross at the $\varphi^{(2)}$ value where the vacuum PFT angle [given by Eq. (4) as a function of propagation] averages to zero in the jet region. The nonzero crossing point and the amplitude asymmetry around the crossing point result from the laser focusing near the downstream end of the gas jet, so the majority of the laser-plasma interaction occurs on one side of laser focus. Naively, one would expect the deflection to increase with larger PFT (and so with larger $\varphi^{(2)}$ ), but Fig. 8 shows the deflection magnitude decreases for $\left|\varphi^{(2)}\right| \gtrsim 500$. The decrease in deflection at larger $\varphi^{(2)}$ is caused by the increasing pulse length and decreasing peak intensity, both of which reduce the wake amplitude and resulting deflection [see Eq. (9)]. The disagreement between theory and experiment is due to the nonlinear conditions of the experiment (PIC simulations predict that the pulse self-focuses to $a_{0} \approx 3$ while the model assumes quasilinear regime), the effect of the plasma interaction on PFT evolution, and the presence of higher order spectral phase in the experimental pulse [23], which changes the relationship between peak power, pulse length, and $\varphi^{(2)}$. Dependence of the laser near-field output mode on $\varphi^{(2)}$ is a possible source of error for the laser pointing determination. Nevertheless, the key features of the model predictions (crossing point, deflection amplitude, dependence on $\varphi^{(2)}$ and $\beta$ ) agree well with the measured trends.

In Fig. 9 the electron beam deflection is plotted for the same values of $\beta$ as in Fig. 8. The amplitude of the deflection rises with the angular dispersion and depends nonlinearly on 
$\varphi^{(2)}$, as with the laser pointing and consistent with the model. Over the range of spatiotemporal coupling scanned in the experiment, we were able to control the final pointing of the electron beam with sub-mrad precision. Simulations show that the electron deflection is sensitive to features smaller than the uncertainty in the gas jet density measurement. Thus, it is not possible to compare the electron pointing in this experiment directly with PIC simulations. Comparison of Figs. 8 and 9 shows that the electrons are deflected relative to the laser and the electron deflection has a different dependance on the PFT. The electrons must thus be steered by a mechanism other that laser deflection, which was predicted by the electron steering model and is consistent with 3D PIC simulations using the code WARPX [22]. Furthermore, the electron energy spectrum was measured with a magnetic spectrometer immediately after the imaging phosphor and was not significantly affected by the addition of $\beta$, except for a decrease in charge, which likely resulted from the drop in laser intensity at focus.

Naively, one expects the electron beam to follow the final laser angle $\theta_{l}$, which is given by integrating Eq. (2) or (9) along the entire laser-plasma interaction length. However, the electron angle relative to the laser $\theta_{e}=(d / d z) x_{e}$ is sensitive to changes in the electron's transverse equilibrium position $x_{E q}$ [Eqs. (10), (11), and (12)] that occur on or faster than the scale of the betatron wavelength $2 \pi k_{\beta}^{-1}$ and so is much more sensitive to the laser evolution and density profile near the end of the gas jet. Any sudden change in the laser or plasma parameters (e.g., the plasma density at the end of an LPA stage) may result in strong electron steering relative to the laser (see Ref. [22]). This simple analysis explains why the approximations provided satisfactory agreement with experimental results for laser steering (integrated), but due to experimental uncertainties the electron steering is too sensitive for predictive simulation and theoretical results.

\section{CONCLUSIONS}

This study proposes a physical interpretation as well as a quantitative model for laser steering and electron beam deflection in asymmetric laser wakefield acceleration, caused by pulse front tilt (PFT) and/or a transverse density gradient. The models show good agreement with particle-in-cell simulations and experiments performed with the BELLA petawatt laser. We show that PFT can routinely result in a $0.1-1 \mathrm{mrad}$ deflection of the electron beam through electron beam steering and/or laser pulse steering in an LPA experiment. With this model, it is possible to set limits on the acceptable PFT in an LPA system for given electron beam performance requirements. Furthermore, this effect can be used to controllably

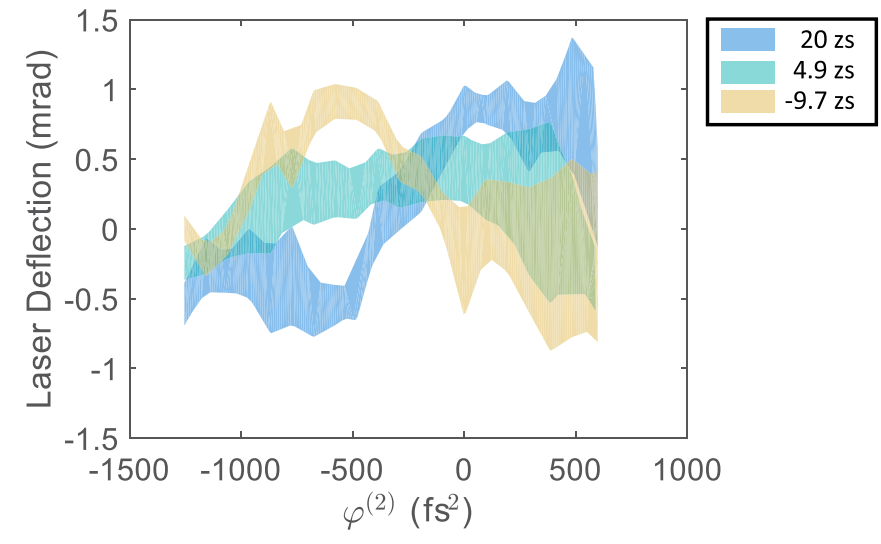

FIG. 10. The effect of laser analysis threshold on laser pointing determination is plotted for $\beta=20,4.9$, and $-9.7 \mathrm{zs}$.

deflect an electron beam at the end of an LPA stage without strongly affecting the electron beam parameters, potentially providing steering control in a high repetition rate LPA and allowing for electron beam pointing feedback and/or stabilization. The ability to deflect the electron beam relative to the output laser direction could also be useful for controlling the stage-to-stage coupling in multistage LPA systems.

\section{ACKNOWLEDGMENTS}

This work was supported by the Director, Office of Science, of the US Department of Energy under Contract No. DE-AC02-05CH11231 as well as the National Science Foundation under Grant No. 1632796. This research used resources of the National Energy Research Scientific Computing Center (NERSC), a US Department of Energy Office of Science User Facility.

\section{APPENDIX: LASER POINTING DETERMINATION}

An intensity threshold was applied to the images of the near-field laser mode (see Fig. 5) to eliminate stray light arising from laser scatter, back-reflections, and laser halo. The results in Fig. 8(a) are sensitive to the intensity threshold used in the laser pointing analysis [40]. The effect of intensity threshold is illustrated in Fig. 10 for a wide range of threshold values, where each colored band represents the range of pointing analysis results for a particular $\beta$. The three bands correspond to the $\beta=20,4.9$, and -9.7 zs plots in Fig. 8(a). Note that the crossing point, the order, and the magnitude of the laser deflection plots in Fig. 10 do not change significantly with threshold.
[1] E. Esarey, C. B. Schroeder, and W. P. Leemans, Rev. Mod. Phys. 81, 1229 (2009).

[2] A. J. Gonsalves, K. Nakamura, J. Daniels, C. Benedetti, C. Pieronek, T. C. H. de Raadt, S. Steinke, J. H. Bin, S. S. Bulanov, J. van Tilborg, C. G. R. Geddes, C. B. Schroeder, C. Tóth, E. Esarey, K. Swanson, L. Fan-Chiang, G. Bagdasarov, N. Bobrova, V. Gasilov, G. Korn, P. Sasorov, and W. P. Leemans, Phys. Rev. Lett. 122, 084801 (2019).
[3] W. P. Leemans, A. J. Gonsalves, H.-S. Mao, K. Nakamura, C. Benedetti, C. B. Schroeder, C. Tóth, J. Daniels, D. E. Mittelberger, S. S. Bulanov, J.-L. Vay, C. G. R. Geddes, and E. Esarey, Phys. Rev. Lett. 113, 245002 (2014).

[4] J. Faure, Y. Glinec, A. Pukhov, S. Kiselev, S. Gordienko, E. Lefebvre, J.-P. Rousseau, F. Burgy, and V. Malka, Nature (London) 431, 541 (2004). 
[5] C. G. R. Geddes, C. Toth, J. Van Tilborg, E. Esarey, C. B. Schroeder, D. Bruhwiler, C. Nieter, J. Cary, and W. P. Leemans, Nature (London) 431, 538 (2004).

[6] S. P. D. Mangles, C. D. Murphy, Z. Najmudin, A. G. R. Thomas, J. L. Collier, A. E. Dangor, E. J. Divall, P. S. Foster, J. G. Gallacher, C. J. Hooker, D. A. Jaroszynski, A. J. Langley, W. B. Mori, P. A. Norreys, F. S. Tsung, R. Viskup, B. R. Walton, and K. Krushelnick, Nature (London) 431, 535 (2004).

[7] W. Leemans and E. Esarey, Phys. Today 62(3), 44 (2009).

[8] H.-P. Schlenvoigt, K. Haupt, A. Debus, F. Budde, O. Jäckel, S. Pfotenhauer, H. Schwoerer, E. Rohwer, J. G. Gallacher, E. Brunetti, R. P. Shanks, S. M. Wiggins, and D. A. Jaroszynski, Nat. Phys. (London) 4, 130 (2008); S. Kneip, C. McGuggey, F. Dollar, M. S. Bloom, V. Chvykov, G. Kalintchenko, K. Krushelnick, A. Maksimchuk, S. P. D. Mangles, T. Matsouka, Z. Najmudin, C. A. J. Palmer, J. Schreiber, W. Schumaker, A. G. R. Thomas, and V. Yanovsky, Appl. Phys. Lett. 99, 093701 (2011).

[9] Z.-H. He, A. Thomas, B. Beaurepaire, J. Nees, B. Hou, V. Malka, K. Krushelnick, and J. Faure, Appl. Phys. Lett. 102, 064104 (2013).

[10] V. Malka, J. Faure, Y. A. Gauduel, E. Lefebvre, A. Rousse, and K. T. Phuoc, Nat. Phys. (London) 4, 447 (2008).

[11] D. Guénot, D. Gustas, A. Vernier, B. Beaurepaire, F. Böhle, M. Bocoum, M. Lozano, A. Jullien, R. Lopez-Martens, A. Lifschitz, and J. Faure, Nat. Phot. (London) 11, 293 (2017).

[12] J. van Tilborg, S. Steinke, C. G. R. Geddes, N. H. Matlis, B. H. Shaw, A. J. Gonsalves, J. V. Huijts, K. Nakamura, J. Daniels, C. B. Schroeder, C. Benedetti, E. Esarey, S. S. Bulanov, N. A. Bobrova, P. V. Sasorov, and W. P. Leemans, Phys. Rev. Lett. 115, 184802 (2015).

[13] A. J. Gonsalves, K. Nakamura, J. Daniels, H.-S. Mao, C. Benedetti, C. B. Schroeder, C. Tóth, J. van Tilborg, D. E. Mittelberger, S. S. Bulanov, J.-L. Vay, C. G. R. Geddes, E. Esarey, and W. P. Leemans, Phys. Plasmas 22, 056703 (2015).

[14] S. P. D. Mangles, A. G. R. Thomas, O. Lundh, F. Lindau, M. C. Kaluza, A. Persson, C.-G. Wahlström, K. Krushelnick, and Z. Najmudin, Phys. Plasmas 14, 056702 (2007).

[15] J. Ferri, X. Davoine, S. Fourmaux, J. C. Kieffer, S. Corde, K. T. Phuoc, and A. Lifschitz, Sci. Rep.-UK 6, 27846 (2016).

[16] B. Beaurepaire, A. Vernier, M. Bocoum, F. Böhle, A. Jullien, J.-P. Rousseau, T. Lefrou, D. Douillet, G. Iaquaniello, R. LopezMartens, A. Lifschitz, and J. Faure, Phys. Rev. X 5, 031012 (2015).

[17] A. J. Gonsalves, K. Nakamura, C. Lin, J. Osterhoff, S. Shiraishi, C. B. Schroeder, C. G. R. Geddes, C. Tóth, E. Esarey, and W. P. Leemans, Phys. Plasmas 17, 056706 (2010).

[18] A. Popp, J. Vieira, J. Osterhoff, Z. Major, R. Hörlein, M. Fuchs, R. Weingartner, T. P. Rowlands-Rees, M. Marti, R. A. Fonseca, S. F. Martins, L. O. Silva, S. M. Hooker, F. Krausz, F. Grüner, and S. Karsch, Phys. Rev. Lett. 105, 215001 (2010).

[19] M. Schnell, A. Sävert, I. Uschmann, M. Reuter, M. Nicolai, T. Kämpfer, B. Landgraf, O. Jäckel, O. Jansen, A. Pukhov, M. C. Kaluza, and C. Spielmann, Nat. Comm. (London) 4, 2421 (2013).

[20] M. C. Kaluza, S. P. D. Mangles, A. G. R. Thomas, Z. Najmudin, A. E. Dangor, C. D. Murphy, J. L. Collier, E. J. Divall, P. S. Foster, C. J. Hooker, A. J. Langley, J. Smith, and K. Krushelnick, Phys. Rev. Lett. 105, 095003 (2010); P. Sprangle, J. Krall, and E. Esarey, ibid. 73, 3544 (1994).
[21] Y. Ma, D. Seipt, S. J. D. Dann, M. J. V. Streeter, C. A. J. Palmer, L. Willingale, and A. G. R. Thomas, Phys. Plasmas 25, 113105 (2018); S. J. E. Brockington, R. D. Horton, D. Q. Hwang, R. W. Evans, S. J. Howard, and Y. C. F. Thio, Rev. Sci. Instrum. 76, 063503 (2005).

[22] M. Thévenet, D. E. Mittelberger, K. Nakamura, R. Lehe, C. B. Schroeder, J.-L. Vay, E. Esarey, and W. P. Leemans, Phys. Rev. Accel. Beams 22, 071301 (2019).

[23] K. Nakamura, H. S. Mao, A. J. Gonsalves, H. Vincenti, D. E. Mittelberger, J. Daniels, A. Magana, C. Toth, and W. P. Leemans, IEEE J. Quantum Electron. 53, 1200121 (2017).

[24] S. Akturk, X. Gu, E. Zeek, and R. Trebino, Opt. Express 12, 4399 (2004); S. Akturk, X. Gu, P. Gabolde, and R. Trebino, ibid. 13, 8642 (2005).

[25] M. Born and E. Wolf, Principles of Optics: Electromagnetic Theory of Propagation, Interference and Diffraction of Light, 7th Edition (Press Syndicate of the University of Cambridge, United Kingdom, 1999), Vol. 461.

[26] T. M. Antonsen, Jr. and P. Mora, Phys. Rev. Lett. 69, 2204 (1992).

[27] L. M. Gorbunov and V. I. Kirsanov, Zh. Eksp. Teor. Fiz. 93, 509 (1987) [Sov. Phys. JETP 66, 290 (1987)].

[28] J.-L. Vay, C. G. R. Geddes, E. Esarey, C. B. Schroeder, W. P. Leemans, E. Cormier-Michel, and D. P. Grote, Phys. Plasmas 18, 123103 (2011).

[29] J.-L. Vay, D. P. Grote, R. H. Cohen, and A. Friedman, Comput. Sci. Disc. 5, 014019 (2012).

[30] J.-L. Vay, Phys. Rev. Lett. 98, 130405 (2007).

[31] J.-L. Vay, A. Almgren, J. Bell, L. Ge, D. Grote, M. Hogan, O. Kononenko, R. Lehe, A. Myers, C. Ng, J. Park, R. Ryne, O. Shapoval, M. Thévenet, and W. Zhang, Nucl. Instrum. Methods A 909, 476 (2018).

[32] C. Benedetti, C. B. Schroeder, C. G. R. Geddes, E. Esarey, and W. P. Leemans, Plasma Phys. Control. Fusion 60, 014002 (2017).

[33] C. Benedetti, C. B. Schroeder, E. Esarey, C. G. R. Geddes, and W. P. Leemans, in Advanced Accelerator Concepts: 14th Advanced Accelerator Concepts Workshop, edited by S. H. Gold and G. S. Nusinovich, AIP Conf. Proc. No. 1299 (AIP, Annapolis, MD, USA, 2010), pp. 250-255.

[34] C. Benedetti, C. B. Schroeder, C. G. R. Geddes, E. Esarey, and W. P. Leemans, in Advanced Accelerator Concepts: 17th Advanced Accelerator Concepts Workshop, edited by S. H. Gold, G. S. Nusinovich, and K. P. Wootton, AIP Conf. Proc. No. 1812 (AIP, National Harbor, MD, USA, 2017), p. 050005.

[35] C. Fiorini, C. Sauteret, C. Rouyer, N. Blanchot, S. Seznec, and A. Migus, IEEE J. Quantum Electron. 30, 1662 (1994).

[36] P. O'Shea, M. Kimmel, X. Gu, and R. Trebino, Opt. Lett. 26, 932 (2001).

[37] G. Pretzler, A. Kasper, and K. J. Witte, Appl. Phys. B 70, 1 (2000).

[38] G. R. Plateau, N. H. Matlis, C. G. R. Geddes, A. J. Gonsalves, S. Shiraishi, C. Lin, R. A. van Mourik, and W. P. Leemans, Rev. Sci. Instrum. 81, 033108 (2010).

[39] P. C. Hansen and M. Saxild-Hansen, J. Comput. Appl. Math. 236, 2167 (2012).

[40] D. E. Mittelberger, Ph.D. thesis, University of California, Berkeley (2017). 